\title{
Primary Adrenal Insufficiency due to Multidrug-Resistant Tuberculosis: A Case Report
}

\author{
Shefin SMa , Hossain $\mathrm{KN}^{\mathrm{b}}$, Ahmed JUc ${ }^{\mathrm{c}}$, Rahman $\mathrm{MM}^{\mathrm{d}}$
}

\begin{abstract}
Tuberculosis (TB) is a common infectious disease in developing countries like Bangladesh. Drug-resistant tuberculosis (DR-TB) poses a significant threat to the population. A relatively uncommon site of involvement of TB is adrenal gland, which is an important cause of chronic adrenal insufficiency. Here we present a case of a 40-year-old male with multidrug-resistant tuberculosis (MDR-TB) involving the adrenal glands. He was previously diagnosed as a case of disseminated TB involving lungs, right knee joint and lumbar spine and received anti-TB chemotherapy for several occasions and in different categories in last 12 years before this presentation. He presented with 4-month history of vomiting, diarrhea, generalized weakness, generalized pigmentation and postural hypotension. Investigations revealed hyponatremia with hyperkalemia. Adrenal insufficiency was suspected and serum cortisol was found to be low and serum adreno corticotropic hormone (ACTH) was high. On imaging there was bilateral adrenal enlargement. Fine needle aspiration cytology (FNAC) was done from adrenal gland which revealed granulomatous lesion suggestive of TB. Gene Xpert and culture-sensitivity of aspirated material for Mycobacterium tuberculosis revealed MDR-TB. Patient was finally diagnosed as a case of primary adrenal insufficiency due to MDR-TB of the adrenal glands and was treated with regime for MDR-TB for 20 months.
\end{abstract}

Key Words: Adrenal insufficiency, adrenal tuberculosis, drug resistant tuberculosis.

(BIRDEM Med J 2017; 7(3): 235-237)

\section{Introduction}

Tuberculosis (TB) may affect many of the endocrine glands including the hypothalamus, pituitary, thyroid and adrenals. The most commonly involved endocrine organ in TB is the adrenal gland. Addison's disease refers to a chronic endocrine disorder characterized by primary adrenal insufficiency. Tuberculous Addison's disease is an important cause of primary adrenocortical insufficiency particularly in the developing countries.

Author Information

a. Dr. Sultana Marufa Shefin, Assistant Professor, Department of Endocrinology, BIRDEM General Hospital, Dhaka, Bangladesh.

b. Dr. Kazi Nazmul Hossain, Registrar, Department of Endocrinology, BIRDEM General Hospital, Dhaka, Bangladesh.

c. Dr. Jamal Uddin Ahmed, Assistant Professor, Department of Medicine, BIRDEM General Hospital, Dhaka, Bangladesh.

d. Dr. Mohammad Mostafizur Rahman, Junior Consultant, Chest Disease Clinic, Noakhali, Bangladesh.

Address of Correspondence: Dr. Sultana Marufa Shefin, Assistant Professor, Department of Endocrinology, BIRDEM General Hospital. Email: shefin_neon@yahoo.com

Received: September 13, 2016

Accepted: July 31, 2017
Recent improvements in imaging techniques and modern biochemical tests for the investigation of adrenal function have given us greater insight into the endocrinology of adrenal tuberculosis. ${ }^{1}$

Multidrug-resistant tuberculosis (MDR-TB) is an emerging and worrisome health problem that threatens TB control worldwide. The diagnosis and clinical management of MDR-TB is a complex issue associated with the use of multiple drugs for a long period, usually apprehended of side effects and high costs. In the past, Mycobacterium tuberculosis (MTB) infection was a major cause of adrenal failure. In the present era however, Addison's disease secondary to primary adrenal TB infection is somewhat rare. ${ }^{2}$ We describe a patient who was diagnosed with Addison's disease secondary to MDR-TB.

\section{Case Report}

A 40-year-old male presented to a private hospital in Dhaka on March 2014 with diarrhea and vomiting for 10 days, generalized weakness, vertigo on changing posture and gradual pigmentation of whole body for 4 months. His past history revealed that he was diagnosed 
as a case of TB of right knee joint in Sheheed Shahrawardy Hospital, Dhaka in August 2003 on the basis of synovial tissue biopsy showing granulomatous inflammation suggestive of TB. Standard dose Anti-TB chemotherapy with 4 drug fixed-dose-combination (4 FDC) was started and was continued beyond 2 months initial phase due to late response. But during treatment he developed back pain and was admitted in Bangabandhu Sheikh Mujib Medical University (BSMMU), where after evaluation he was diagnosed as a case of Pott's disease. Here, along with ongoing 4FDC, injection Streptomycin was added on December 2003 and it was given for 2 months. After that 4-FDC was continued without switching to 2 drug fixed dose combination (2-FDC) probably due to delayed response (documents available). Without significant improvement patient went abroad to India (Kolkata) in August 2004 where he was diagnosed as a case of new smear negative pulmonary TB (PTB) along with previous diagnosis and the ongoing 4-FDC was continued without further modification of treatment or evaluation. In September 2004 he was admitted in a private medical college in Dhaka where he was suspected as a case of multi-drug resistant TB (MDR-TB) but further evaluation in this respect was not done. In July 2005 he developed cold abscess in right thigh which was surgically evacuated. Following that he was improving and stepped down to 2-FDC and ultimately anti-TB was stopped in March 2006 (after total 2 years and 7 months) when he was declared clinically cured. He was asymptomatic for next 3 years, till April 2010 when he again developed arthritis of right knee joint and this time he was admitted in National Institute of Traumatology, Orthopedics and Rehabilitation (NITOR), Dhaka and diagnosed as tubercular arthritis of right knee. Standard dose antiTB drug (category-I) was started but it was modified by replacing ethambutol with levofloxacin as he developed retrobulbar neuritis. He received this regimen for a total 18 months and was cured.

Subsequently he was doing well till March 2014 when he presented to a private hospital during this episode of illness. On examination, patients pulse was 110 beats/ min, blood pressure was $80 / 50 \mathrm{~mm}$ of $\mathrm{Hg}$, and he was severely dehydrated. Initial laboratory investigations showed- serum sodium- $125 \mathrm{mmol} / \mathrm{l}$, potassium 6.9 $\mathrm{mmol} / \mathrm{l}$, S. creatinine- $2.3 \mathrm{mg} / \mathrm{dl}$. Serum basal cortisol was low (1.0 microgm/dl). ACTH level was $131 \mathrm{pmol} /$ L. Rapid ACTH stimulation test revealed serum cortisol $5.62 \mathrm{microgm} / \mathrm{dl}$ at 30 minutes and $8.34 \mathrm{microgm} / \mathrm{dl}$ at 60 minutes after $250 \mathrm{1} / \mathrm{g}$ g Synacthen injection. Ultrasonogram (USG) of whole abdomen showed right adrenal enlargement. Computed tomography (CT) scan of abdomen showed bilateral adrenal mass. CT guided FNAC was done from right adrenal gland which revealed granulomatous inflammation suggestive of TB. The aspirated material was sent for Gene X-pert and acid fast bacilli (AFB) culture-sensitivity that revealed growth of Mycobacterium tuberculosis resistant to both isoniazid and rifampicin. Patient was diagnosed as a case of primary adrenal insufficiency due to MDR-TB of the adrenal glands and was referred to MDR-TB treatment center in National Institute of Diseases of Chest and Hospital (NIDCH) for further management. There he received anti-TB chemotherapy for MDR-TB for a total of 20 months. For his adrenal insufficiency, after initial management with intravenous (IV) hydrocortisone, patient was switched to oral prednisolone without development of any adrenal crisis during the treatment period. Patient needed higher dose of oral prednisolone, $10 \mathrm{mg}$ at morning and $5 \mathrm{mg}$ at evening during the period of anti-TB treatment. After the completion of the course of MDR-TB treatment regimen patient was declared as cured and USG showed regression of enlarge adrenal glands.

\section{Discussion}

TB has once again become one of the major global public health care concerns. ${ }^{3}$ Over two billion people are infected today by MTB, leading to approximately 5,000 deaths every day. ${ }^{4}$ Interestingly, approximately $80 \%$ of the newly diagnosed cases occur in high-TB burdened countries, mainly in Asia and Africa. ${ }^{5}$ These places are at increased risk from drug-resistant strains due to limited access to medical attention, delay in diagnosis and chemotherapy initiation and as a result of low therapeutic adherence. Approximately 0.5 million of MDR-TB cases are diagnosed per year; however, this number is too optimistic as less than $7 \%$ are reported to the World Health Organization (WHO). ${ }^{6}$

TB is a common cause of primary adrenal insufficiency in the world. Clinically significant adrenal insufficiency appears to occur in about $5 \%$ of patients with disseminated TB. ${ }^{7}$ Tuberculous Addison's disease results from hematogenous spread of the infection from elsewhere in the body; extra-adrenal disease is usually evident. The adrenals are initially enlarged, with extensive epithelioid granulomas and caseation and both the cortex and the medulla are affected. Fibrosis ensues and the adrenals become normal or smaller in size, with calcification evident in $50 \%$ of cases. ${ }^{8}$ Adrenal involvement was found in $6 \%$ of patients with active TB in an autopsy series. ${ }^{9}$ More than $90 \%$ of the gland 
must be destroyed before insufficiency appears. The majority of patients with active or recently acquired disease $(<2$ years) have bilateral adrenal enlargement, while calcification and atrophy are the norm with more remote infection or inactive disease. ${ }^{10}$ With the use of anti-TB chemotherapy, it may even be reversible if detected in early stages. Rifampicin may accelerate the metabolic clearance of cortisol, thereby increasing the replacement dose of corticosteroid needed in these patients. ${ }^{7}$

In this case, there is presence of adrenal insufficiency due to MDR-TB affecting the adrenal glands, proven by clinical, biochemical, radiological and microbiological investigations. There is scarce data regarding MDR-TB causing adrenal insufficiency. One study carried out in patients with MDR-TB showed a 49.5\% prevalence of adrenocortical dysfunction by using the high-dose $(250 \mathrm{mg})$ ACTH stimulation test. ${ }^{11}$ In another study on patients with MDR-TB using the low-dose ACTH stimulation test, only $5-10 \%$ prevalence of adrenal insufficiency was found. At follow-up, after the intensive phase of anti-TB treatment, the cortisol response was restored in all cases. ${ }^{12}$ It is to be noted that although there was biochemical evidence of adrenal insufficiency, microbiological evidence of MTB affecting the adrenal glands was not available in these studies. In some of the cases, radiological evidence of adrenal enlargement was found. But the fact that the adrenals can be enlarged in patients with pulmonary tuberculosis without active involvement of the glands has been demonstrated in various studies. Stress and inflammation could be the potential reasons behind it. ${ }^{13}$ Therefore, our case is unique in this respect that it shows the direct involvement of the adrenal glands by MDR-TB.

\section{Conclusion}

MDR-TB is an important cause of morbidity and mortality, especially in the developing countries like Bangladesh. Co-existing adrenal insufficiency, which is often underdiagnosed, will further aggravate the situation. Our case may only show the tip of the iceberg. Nationwide large scale studies may therefore be undertaken to screen for adrenal insufficiency in MDRTB patients to discover the actual magnitude of the problem. Also, health personnel caring for MDR-TB patients should hold a high degree of suspicion regarding development of adrenal insufficiency in these patients and take prompt and appropriate measures when suspicion arises.

\section{Conflict of interest: None}

\section{References}

1. Bhatia E, Jain SK, Gupta RK, Pandey R. Tuberculous Addison's disease: Lack of normalization of adrenocortical function after anti-tuberculous chemotherapy. Clin Endocrinol 1998; 48 (3): 355-59.

2. Brandao RA, Carvalho JF. Diagnosis and classification of Addison's disease (autoimmune adrenalitis). Autoimmun Rev 2014; 13(4-5): 408-11.

3. Vynnycky E, Fine PEM. Interpreting the decline in tuberculosis: the role of secular trends in effective contact. Int J Epidemiol 1999; 28(2): 327-34.

4. World Health Organization (2007). Tuberculosis Fact Sheet. Fact Sheet No.104. Available: http://www.who.int/ mediacentre/ factsheets/fs104/en/print. html. Accessed 25 September 2007.

5. Parsons LM, Somoskövi A, Gutierrez C, Lee E, Paramasivan $\mathrm{CN}$, Abimiku A et al. Laboratory diagnosis of tuberculosis in resource-poor countries: challenges and opportunities. Clin Microbiol Rev 2011; 24(2): 314-50.

6. World Health Organization, Global Tuberculosis Report 2014, World Health Organization, Geneva,Switzerland,2014, http:/ /apps.who.int/iris/bitstream/10665/137094/ 1/ 9789241564809_eng.pdf. Acessed.

7. Caroll TB, Aron DC, Findling JW, Tyrell JB. Glucocorticoids and Adrenal Androgens. In: Gardner DG, Shoback D, eds. Greenspan's Basic \& Clinical Endocrinology. $9^{\text {th }}$ ed. New York: The McGraw-Hill Companies. 2012: P.-305.

8. Stewart PM, Krone NP. The Adrenal Cortex. In: Melmed S, Polonsky KS, Larsen PR, Kronenberg HM, eds. Williams Textbook of Endocrinology 12 ed. Philadelphia: Saunders, 2011: P.-516.

9. Lam KY, Lo CY. A critical examination of adrenal tuberculosis and a 28-year autopsy experience of active tuberculosis. Clin Endocrinol 2001; 54(5): 633-39.

10. Kelestimur F. The endocrinology of adrenal tuberculosis: The effects of tuberculosis on the hypothalamo-pituitaryadrenocortical function. J Endocrinol Invest 2004; 27(4): $380-86$.

11. Prasad GA, Sharma SK, Mohan A. Adrenocortical reserve and morphology in tuberculosis. Indian J Chest Dis Allied Sci 2000; 42(2): 83-93.

12. Rodríguez-Gutiérrez R, Rendon A, Barrera-Sánchez M, Carlos-Reyna KE, Álvarez-Villalobos NA, González-Saldivar $\mathrm{G}$ et al. Multidrug-Resistant Tuberculosis and Its Association with Adrenal Insufficiency: Assessment with the Low-Dose ACTH Stimulation Test. Int J Endocrinol 2016; doi: 10.1155/ 2016/9051865. Accessed (date)

13. Upadhyay J, Sudhindra P, Abraham G, Trivedi N. Tuberculosis of the adrenal gland: A case report and review of the literature of infections of the adrenal glands. Int J Endocrinol 2014; doi.org/10.1155/2014/876037. Accessed (date) 